

\title{
Driving competition in a complex ecosystem: application to anaerobic digestion
}

Pierre Masci, Olivier Bernard, Frédéric Grognard, Eric Latrille, Jean-Baptiste Sorba, Jean-Philippe Steyer

\section{- To cite this version:}

Pierre Masci, Olivier Bernard, Frédéric Grognard, Eric Latrille, Jean-Baptiste Sorba, et al.. Driving competition in a complex ecosystem: application to anaerobic digestion. European Control Conference, Aug 2009, Budapest, Hungary. hal-01091826

\section{HAL Id: hal-01091826 \\ https://hal.inria.fr/hal-01091826}

Submitted on 8 Dec 2014

HAL is a multi-disciplinary open access archive for the deposit and dissemination of scientific research documents, whether they are published or not. The documents may come from teaching and research institutions in France or abroad, or from public or private research centers.
L'archive ouverte pluridisciplinaire HAL, est destinée au dépôt et à la diffusion de documents scientifiques de niveau recherche, publiés ou non, émanant des établissements d'enseignement et de recherche français ou étrangers, des laboratoires publics ou privés. 


\title{
Driving competition in a complex ecosystem: application to anaerobic digestion
}

\author{
P. Masci, O. Bernard, F. Grognard, E. Latrille and J.-B. Sorba and J.P. Steyer
}

\begin{abstract}
Anaerobic digestion is a wastewater treatment process where bacteria degrade an organic substrate and produce methane, which can be used as a biofuel. The first task when starting up an anaerobic digester is the increase of its microbial population. It is a delicate phase, which is still not well understood, and its influence on the digester's future performance is not well known. During this phase, we show that a competition between the various species occurs and finally some species become dominant. In this paper, extending the competitive exclusion principle, we propose to drive the competition during this start-up phase, by regulating the volatile fatty acids concentration, with the aim of selecting species with good performance in the standard operating mode of the process. This new "selective" start-up strategy should lead to more efficient ecosystems.

Keywords: Anaerobic digestion; Bioreactors;Biological systems; Competition; Selection; Directed Evolution
\end{abstract}

\section{INTRODUCTION AND MOTIVATION}

Anaerobic digestion is a more and more widely used bioprocess for wastewater treatment. This complex ecosystem involves several hundreds of bacterial and achaeal species [1] that progressively degrade organic matter into methane and $\mathrm{CO}_{2}$. It has many advantages compared to the more widespread activated sludge process: it can handle concentrated substrates, produces few sludge, and methane can be recovered and used as a biofuel. However this process is difficult to manage since the steady state associated to the operating mode is not globally stable [2]. As a corollary, the startup of the digester is a long and risky phase [3] during which the digester loading is progressively increased in order to let the bacterial population adapt, grow and settle the reactor. Reactor startup is then a long procedure (from one month to almost one year) that is essential to achieve a high treatment capacity at steady-state [4], and therefore this phase should be better understood and controlled.

Despite its key role, this phase did not receive so far much attention, perhaps because it is quite

P. Masci, O. Bernard and F. Grognard are with INRIA Sophia Antipolis, 2004 route des lucioles, BP 93, 06902 Sophia Antipolis, France olivier.bernardeinria.fr

J.B. Sorba, E. Latrille and J.P. Steyer are with LBE, UR050, INRA, Avenue des étangs 11100 Narbonne, France difficult to properly characterise since biomass measurements are not available. In [5], it was shown that disturbing the microbial community in a "bang bang" like approach leads to better diversity and thus improves the bioprocess when facing a toxicant.

In this paper, we propose a new model for the start-up phase, including biodiversity. We assume that, at the beginning, $N$ species are competing for the substrate. We show that, depending on the way the digester is started up, some populations will be enhanced and will preferentially remain in the digester. The underlying idea relies on the competitive exclusion principle, which has been theoretically studied and experimentally demonstrated in various conditions [6], [7]. We exploit the idea proposed in [8] to drive the competition during the start-up phase, by regulating the volatile fatty acids (VFA) concentration around a fixed value, which is determined by the normal operating mode of the digester. By doing so, we act upon the ecosystem in order to enhance the process efficiency. Also, bacterial biomass extinction, which is one of the main risks of the start-up phase, is avoided, and the obtained ecosystem presents an interesting steadiness property. Simulations and a first experiment are presented for supporting this start-up strategy.

\section{PRocess MODELLING}

\section{A. Presentation of the model}

We propose here a simple macroscopic model (derived from [9]) in order to be able to handle the involved mathematics. We assume that, in a first acidogenesis step, the dissolved organic substrate, of concentration $s_{1}$, is degraded by a population made of $N_{a}$ species of acidogenic bacteria $\left(y_{j}\right.$, with $j \in\left[1 ; N_{a}\right]$ ) into volatile fatty acids (VFA, denoted $\left.s_{2}\right)$. The growth rate of these bacteria is $\gamma_{j}\left(s_{1}\right)$ :

$$
k_{1} s_{1} \stackrel{\gamma_{j}\left(s_{1}\right) y_{j}}{\longrightarrow} y_{j}+k_{2} s_{2}+k_{5} \mathrm{CO}_{2}
$$

where $k_{1}, k_{2}$ and $k_{5}$ are pseudo-stoichiometric coefficients which represent the transfer from substrate to acidogenic biomass, VFA and $\mathrm{CO}_{2}$. They are assumed to be constant between the various acidogenic species. 
In a second step (methanogenesis), the VFA are degraded into $\mathrm{CH}_{4}$ and $\mathrm{CO}_{2}$ by $N_{m}$ methanogenic archaeabacteria $\left(x_{i}\right.$, with $\left.i \in\left[1 ; N_{m}\right]\right)$ with growth rate $\mu_{i}\left(s_{2}\right)$ :

$$
k_{3} s_{2} \stackrel{\mu_{i}\left(s_{2}\right) x_{i}}{\longrightarrow} x_{i}+k_{4} \mathrm{CH}_{4}+k_{6} \mathrm{CO}_{2}
$$

where $k_{3}, k_{4}$ and $k_{6}$ represent the transfer from substrate to methanogenic biomass, methane and $\mathrm{CO}_{2}$. They are also assumed to be constant between all the methanogenic species.

We assume that the concentrations are perfectly distributed throughout the reactor, and the dilution rate for the dissolved components is $D$. Since a part of the bacteria are attached on a fixed biofilm within the digester, the dilution rate for the $y_{j}$ and $x_{i}$ biomasses are $\beta_{j} D$ and $\alpha_{i} D$ (i.e. the biomasses have a retention time $1 /\left(\beta_{j} D\right)$ or $\left.1 /\left(\alpha_{i} D\right)\right)$. The dynamical mass-balance model in a continuous stirred tank reactor is then straightforwardly derived [10], [9]:

$$
\left\{\begin{aligned}
\dot{s}_{1}= & D\left(s_{1}^{i n}-s_{1}\right)-k_{1} \sum_{j=1}^{N_{a}} \gamma_{j}\left(s_{1}\right) y_{j} \\
\dot{y}_{j}= & \gamma_{j}\left(s_{1}\right) y_{j}-\beta_{j} D y_{j} \\
\dot{s}_{2}= & D\left(s_{2}^{i n}-s_{2}\right)+k_{2} \sum_{j=1}^{N_{a}} \gamma_{j}\left(s_{1}\right) y_{j} \\
& -k_{3} \sum_{i=1}^{N_{m}} \mu_{i}\left(s_{2}\right) x_{i} \\
\dot{x}_{i}= & \mu_{i}\left(s_{2}\right) x_{i}-\alpha_{i} D x_{i} \\
q_{m}= & k_{4} \sum_{i=1}^{N_{m}} \mu_{i}\left(s_{2}\right) x_{i}
\end{aligned}\right.
$$

where $s_{1}^{i n}$ and $s_{2}^{i n}$ are respectively the concentration of the influent organic substrate and influent VFA, and $q_{m}$ is the methane flow rate.

We consider a Monod kinetics (2) for the substrate-saturated growth rate of acidogenic bacteria

$$
\gamma_{j}\left(s_{1}\right)=\bar{\gamma}_{j} \frac{s_{1}}{s_{1}+h_{j}^{1}}
$$

and an Haldane function (3) for the methanogenesis to represent the possible inhibition by an accumulation of VFA [9]:

$$
\mu_{i}\left(s_{2}\right)=\bar{\mu}_{i} \frac{s_{2}}{s_{2}+h_{i}^{2}+\left(s_{2} / h_{i}^{2 *}\right)^{2}}
$$

where $\bar{\gamma}$ is the maximal growth rate of the acidogenic bacteria and $\bar{\mu}$ is the potential maximum growth rates of the methanogenic bacteria, $h_{j}^{1}$ and $h_{i}^{2}$ the half-saturation constants associated to substrates $s_{1}$ and $s_{2}$, and $h_{i}^{2 *}$ the inhibition constants associated to substrate $s_{2}$.

\section{THE "SELECTIVE" START-UP STRATEGY}

\section{A. Standard start-up strategy}

The main danger during the start-up phase is imposing too high a dilution rate $D$, such that the VFA concentration becomes high and inhibitory, and all the methanogenic biomasses are washed out of the digester (see [2] for a study of the two stable equilibria attraction bassins, in the mono-specific case). In order to avoid such a dramatic scenario, the plant operator often starts the digester with a very low dilution rate. Under such a control, the outcome of competition between bacterial species can be predicted in the case where $\forall j \in\left\{1, \cdots, N_{a}\right\}, \beta_{j}=\beta$ and $\forall i \in\left\{1, \cdots, N_{m}\right\}, \alpha_{i}=\alpha$ for some $\alpha, \beta>0$.

Any acidogenic species whose maximum growth rate $\bar{\gamma}_{j}$ is lower than the dilution rate $\beta D$ will be excluded from the digester, because for such a species $\dot{y}_{j}<-K_{j} y_{j}$, with $K_{j}=-\bar{\gamma}_{j}+\beta D>$ 0 , and then $y_{j}(t)<y_{j}(0) e^{-K_{j} t}$ tends to zero. The same exclusion applies for all methanogenic species such that $\max _{s_{2}} \mu_{i}\left(s_{2}\right)<\alpha D$.

For the other acidogenic species, it is possible to define equilibrium substrate concentration $s_{1 j}^{*}$ such that

$$
\gamma_{j}\left(s_{1 j}^{*}\right)=\beta D
$$

where the $s_{1 j}^{*}$ concentration depends on $D$ :

$$
\frac{\partial}{\partial D} s_{1 j}^{*} \geq 0
$$

For the other methanogenic species, it is possible to define $s_{2 i}^{*}$ and $s_{2 i}^{\dagger} \geq s_{2 i}^{*}$ such that

$$
\mu_{i}\left(s_{2 i}^{*}\right)=\mu_{i}\left(s_{2 i}^{\dagger}\right)=\alpha D
$$

where the $s_{2 i}^{*}$ and $s_{2 i}^{\dagger}$ concentrations depend on $D$ :

$$
\frac{\partial}{\partial D} s_{2 i}^{*} \geq 0 \text { and } \frac{\partial}{\partial D} s_{2 i}^{\dagger} \leq 0
$$

In order to prove the next property, we can then define a dilution rate $D^{*}$ low enough such that:

$$
\min _{i}\left(s_{2 i}^{\dagger}\right)=\max _{i}\left(s_{2 i}^{*}\right)
$$

and, for any $S>0$, there exists $D^{S}<D^{*}$ such that $\forall D<D^{S}$,

$$
\min _{i}\left(s_{2 i}^{\dagger}\right)>S>\max _{i}\left(s_{2 i}^{*}\right)
$$

The following property determines the outcome of the competition when the dilution is small enough and when there is enough substrate in the input to avoid the straightforward wash-out of all species:

Property: If $\forall j, \beta_{j}=\beta, \forall i, \alpha_{i}=\alpha$, the dilution satisfies $D<D^{*}$, and $s_{1}^{i n}>\min _{j}\left(s_{1 j}^{*}\right)$ then

- the species with lowest $s_{1 j}^{*}$ is selected among all acidogenic species (with $\lim _{t \rightarrow \infty} y_{j}=y_{j}^{*}$ ), if, moreover, $S=s_{2}^{i n}+\frac{k_{2}}{k_{1}}\left(s_{1}^{i n}-s_{1 j}^{\star}\right)$ satisfies (4)

- the species with lowest $s_{2 i}^{*}$ is selected among all the methanogenic species.

Proof : Under such a constant low dilution rate, the theory of competitive exclusion predicts (with 
Monod-like growth functions) that the most efficient species for the growth $\beta D$, i.e. the acidogenic species with lowest $s_{1 j}^{*}$, is selected [6]. This is also true with Haldane-like growth functions when (4) is satisfied [11]: the methanogenic species with lowest $s_{2 i}^{*}$ wins the competition and excludes all others.

Then if a constant dilution rate $D<D^{*}$ is imposed, two selection processes occur, which do not depend on the $s_{1}^{i n}$ and $s_{2}^{i n}$ input concentrations. Note that these two processes are independent, and that the trait which is optimized is the lowest substrate requirement. It is not possible to choose the $s_{1 j}^{*}$ and $s_{2 i}^{*}$ concentrations: selection occurs but it is not controlled. That is a major problem of this start-up strategy: species which would grow fast in the standard operating mode can be washed out; uncontrolled competition can lead to the selection of species with low growth rate in the standard operating mode, thus slowing the pollution removal process.

We propose an alternative strategy which permits to control the competition and select the most efficient species in the standard operating mode.

\section{B. Objective of the proposed strategy}

A reasonable objective for an anaerobic digester is to consume the pollutant substrate and obtain a constant VFA output concentration $\bar{s}_{2}$ (VFA is necessary for the process, but it is also a pollutant), with the highest possible dilution rate $D$, which corresponds to the wastewater treatment rate.

The objective of the strategy that we want to explore is to preserve and select the species whose equilibrium $\mu_{i}\left(\bar{s}_{2}\right) / \alpha_{i}$ (denoted "relative growth rate") is maximal, thus optimizing the wastewater treatment rate (at equilibrium $D=\mu_{i}\left(\bar{s}_{2}\right) / \alpha_{i}$ ). More than that, we propose to drive this selection since the beginning of the start-up phase, which may have a positive impact on the biofilm composition, thus resulting in better performances. For achieving this goal we regulate $s_{2}$ and show that it leads to the desired competition outcome.

\section{Control design for the regulation of $s_{2}$}

Here we assume that a control strategy has been set-up which ensures the convergence of $s_{2}$ towards the setpoint $\bar{s}_{2}<s_{2}^{i n}$. Because of space limitation, we won't detail the considered control law and neither prove its convergence. However we refer the reader to other works aiming at the regulation of substrates in an anaerobic digestion process [10], [12], [13]. In the example section, the used controller is presented and its convergence is shown on a real plant.

\section{Selection of archaeal species with maximal} $\mu_{i}\left(s_{2}\right) / \alpha_{i}$

The principle of driving the competition for selecting species or individuals which maximize a chosen criterion is presented in [8], where a general law is given for this selection, and specific selection criteria are given for Monod and Droop models. This theory can also be adapted and applied to system (1) if the VFA concentration is regulated to a constant value.

Main Theorem : In system (1), if a controller achieves the regulation of $s_{2}$ to $\bar{s}_{2}<s_{2}^{i n}$, then the methanogenic species $x_{k}$ with highest relative growth rate $\mu_{k}\left(\bar{s}_{2}\right) / \alpha_{k}$ is selected and all the others are excluded.

Proof In a first step we will prove that the regulation of $s_{2}$ to $\bar{s}_{2}<s_{2}^{\text {in }}$ leads to the boundedness of the total methanogenic biomass $\sum_{i=1}^{N_{m}} x_{i}(t)$ (denoted $\left.X_{T}(t)\right)$ in $\left[X_{0} ; X_{m}\right]$ with $X_{0}>0$. Therefore the total methanogenic biomass $X_{T}$ cannot diverge, and its complete washout cannot occur (at least one methanogenic species will remain in the chemostat). In a second step, we will show that the difference in relative velocity between species lead to the survival of only one species, with maximal relative growth rate.

First, $s_{2}$ regulation causes the total methanogenic biomass boundedness. Indeed, defining the variable $z=s_{1}+\frac{k_{1}}{k_{2}} s_{2}+\frac{k_{3} k_{1}}{k_{2}} X_{T}$ and $z^{i n}=s_{1}^{i n}+\frac{k_{1}}{k_{2}} s_{2}^{i n}$, we have

$$
\dot{z}=D z^{i n}-D s_{1}-\frac{k_{1}}{k_{2}} D s_{2}-D \frac{k_{3} k_{1}}{k_{2}} \sum_{i} \alpha_{i} x_{i}
$$

so that

$$
D\left(z^{i n}-z\right) \leq \dot{z} \leq D\left(z^{i n}-\alpha z\right)
$$

where $\alpha=\min _{i} \alpha_{i}$. We directly conclude that an upperbound on $X_{T}$ is deduced from an upperbound on $z$, so that

$$
X_{m}=\frac{k_{2}}{k_{3} k_{1}} \max \left(\frac{z^{i n}}{\alpha}, z(0)\right)
$$

We can deduce from this upperbound that $\int_{0}^{\infty} D(\tau) d \tau$ is unbounded. Indeed, if it was not the case and knowing that $s_{2}$ converges to $\bar{s}_{2}$, any $\dot{x}_{i}$ equation aymptotically yields

$$
\dot{x}_{i}=\left(\mu\left(\bar{s}_{2}\right)-D(\tau)\right) x_{i}
$$

whose solution is unbounded when the aforementionned integral is bounded, which is in contradiction with the existence of $X_{m}$.

Also, the unboundedness of $\int_{0}^{\infty} D(\tau) d \tau$ and (5) yield $\liminf \operatorname{in}_{t \rightarrow \infty} z \geq z^{i n}$, so that, having $\lim _{t \rightarrow \infty} s_{2}=\bar{s}_{2}$ and using the fact that $s_{1}(t) \leq s_{1}^{i n}$ for all times

$$
\liminf _{t \rightarrow \infty} X_{T} \geq \frac{1}{k_{3}}\left(s_{2}^{i n}-\bar{s}_{2}\right)
$$


so that there is a lower-bound $X_{0}$ on $X_{T}$.

Secondly, $X_{T}$ being lower and upper bounded, let us show that the difference in relative growth rates between the species lead to competitive exclusion. We denote $x_{k}$ the species with maximal $\mu_{k}\left(\bar{s}_{2}\right) / \alpha_{k}$, such that $\mu_{k}\left(\bar{s}_{2}\right) / \alpha_{k}>$ $\mu_{i}\left(\bar{s}_{2}\right) / \alpha_{i}, \forall i \neq k$ and $t_{s}$ a time such that $\mu_{k}\left(s_{2}(t)\right) / \alpha_{k}>\mu_{i}\left(s_{2}(t)\right) / \alpha_{i}, \forall i \neq k, \forall t \geq t_{s}$. Let us define

$$
r_{i}=\ln \left(\frac{x_{k}^{1 / \alpha_{k}}}{x_{i}^{1 / \alpha_{i}}}\right)
$$

which leads to

$\dot{r}_{i}(t)=\frac{\dot{x}_{k}}{\alpha_{k} x_{k}}-\frac{\dot{x}_{i}}{\alpha_{i} x_{i}}=\frac{\mu_{k}\left(s_{2}(t)\right)}{\alpha_{k}}-\frac{\mu_{i}\left(s_{2}(t)\right)}{\alpha_{i}}>0$

for all $t \geq t_{s}$. Then $r_{i}(t)$ will increase, so that $\lim _{t \rightarrow+\infty} r_{i}(t)=+\infty$. This means that the ratio $\frac{x_{k}^{1 / \alpha_{k}}}{x_{i}^{1 / \alpha_{i}}}$ will tend to infinity.

As $x_{k}$ is upper bounded by $X_{m}$, it cannot diverge, which implies that

$$
\lim _{t \rightarrow+\infty} x_{i}(t)=0, \forall i \neq k
$$

As $x_{k}$ is also lower bounded by $X_{0}$, then

$$
\liminf _{t \rightarrow+\infty} x_{k}(t)>0
$$

and we obtain that only methanogenic species $x_{k}$ is not excluded from the chemostat.

Thus, if one can regulate the VFA concentration $s_{2}$ so that it remains constant at a given $\bar{s}_{2}$ value, then the selection of the species with maximal relative growth rate $\mu_{k}\left(\bar{s}_{2}\right) / \alpha_{k}$ will occur, thus shaping the ecosystem and optimizing the wastewater treatment rate at equilibrium $D=\mu_{k}\left(\bar{s}_{2}\right) / \alpha_{k}$.

\section{Simulations}

Even though, as previously said, several hundreds of microbial species are present in anaerobic digesters, we have simulated system (1) with only 3 acidogenic species and 4 methanogenic species to keep the system understandable. Species-specific parameters are taken from [9] with $\pm 50 \%$ values. The substrate and VFA inputs were $s_{1}^{\text {in }}=$ $5 \mathrm{~g} / \mathrm{L}$ and $s_{2}^{i n}=5 \mathrm{mmol} / \mathrm{L}$. The relative growth functions $\mu_{i}\left(s_{2}\right) / \alpha_{i}$ of the various methanogenic species is presented in Fig. 1. Species 1 grows better with low VFA concentration, species 3 grows faster under high VFA concentration, and species 2 is better for medium ones. Species 4 is less efficient than the others for any VFA concentration, so that it should be excluded whatever the start-up strategy.

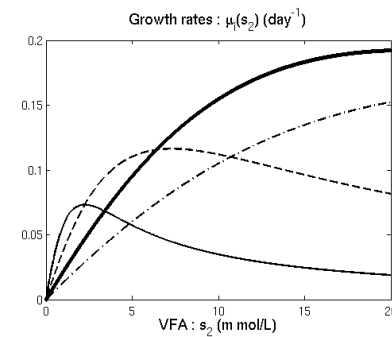

Fig. 1. Relative growth functions $\mu_{i}\left(s_{2}\right) / \alpha_{i}$ of the 4 methanogenic species used in simulation. Species 1 (Solid), species 2 (Dashed), species 3 (Thick), species 4 (Dashdot)

\section{A. Interest of the "selective" start-up strategy}

In this section we show the interest of the selective start-up strategy, by comparing it with two other strategies.

The first strategy is the standard start-up strategy where $D$ is chosen low $(D=0.05)$ to avoid bacterial wash out. The result is presented in Fig. 2 , where we can see that species 1 , which grows slowly for high VFA concentrations, is selected. The digester was started safely but the obtained ecosystem is such that the digester's performance will not be optimal.

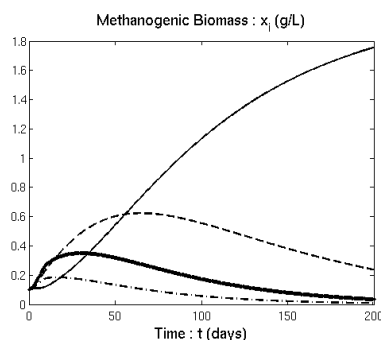

Fig. 2. Standard start-up strategy with low $D=0.05$, to avoid archaeal washout. This strategy leads to the selection of species 1 , which is not optimal for VFA concentrations higher than $2 \mathrm{mmol} / \mathrm{L}$ (see Fig. 1)

The second strategy is the selective start-up strategy where the species which grows faster at VFA concentration $\bar{s}_{2}$ is favored. In Fig. 3 we use a controller to regulate $s_{2}$ to $\bar{s}_{2}=3 \mathrm{mmol} / \mathrm{L}$, and species 2 is selected. We can see on Fig. 1 that it is the most efficient species at concentration $3 \mathrm{mmol} / \mathrm{L}$.

For this strategy we used the following pseudolinearizing controller

$D=\frac{\lambda_{1}\left(\bar{s}_{2}-s_{2}\right)+\frac{k_{3}}{k_{4}} q_{m}}{s_{2}^{i n}-s_{2}}+\lambda_{2} \int_{0}^{t}\left(\bar{s}_{2}-s_{2}(s)\right) d s$

( $\lambda_{1}$ and $\lambda_{2}$ are two gains) where the non-measured acidogenic activity is replaced by a PI correction.

The last one is a naive start-up strategy where the value of $D$ is constant and equal to $\mu_{2}\left(\bar{s}_{2}\right)$. 


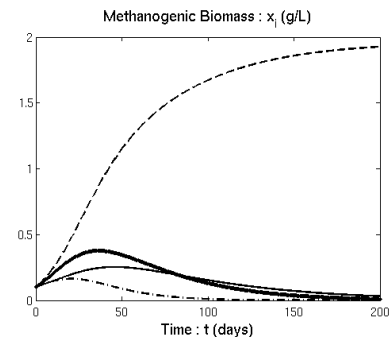

Fig. 3. Selective start-up strategy : species 2, which is the most efficient under VFA concentration $\bar{s}_{2}=3 \mathrm{mmol} / \mathrm{L}$, is selected.

The objective of this strategy is to obtain the same result as in the selective strategy, but the simulation of Fig. 4 shows that it leads to process failure: the VFA were accumulated such that they became inhibitory $(\approx 100 \mathrm{mmol} / \mathrm{L})$, and the methanogenic biomasses were washed out.

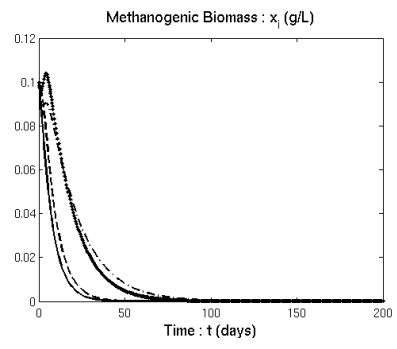

Fig. 4. Naive start-up strategy : by choosing $D$ high $(D=$ $\mu_{2}\left(\bar{s}_{2}\right)$ ) since the beginning of start-up, the methanogenic biomasses became inhibited and were washed out.

These simulations show that the selective startup strategy avoids bacterial extinction, while selecting efficient species for the digester in the standard operating mode.

\section{B. Selecting a population optimal for a given VFA concentration}

Let us suppose now that we want an anaerobic digester whose VFA concentration in the standard operating mode is $\tilde{s}_{2}=8 \mathrm{mmol} / \mathrm{L}$. Using linearizing controller (6) to regulate $s_{2}$ to $\tilde{s}_{2}$, we obtain the simulation of Fig. 5 where species 3 (the most appropriate at concentration $8 \mathrm{mmol} / \mathrm{L}$ ) is selected. This simulation emphasizes that depending on the digester VFA concentration in the standard operating mode, different species should be selected during the start-up phase, so that the obtained ecosystem will be more efficient.

\section{The selective process consolidates the ecosys- tem}

On Fig. 6 we can see that the dilution rate, during the $\tilde{s}_{2}$ selective start-up, rises until selection

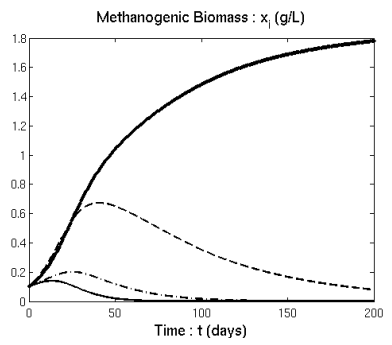

Fig. 5. Start-up selection, under the closed loop control, of species which maximize $\mu_{2}\left(\tilde{s}_{2}\right) / \alpha_{i}$, with $\tilde{s}_{2}=8 \mathrm{mmol} / \mathrm{L}$. Depending on the VFA concentration in the standard operating mode, different populations should be selected to optimize the process.

occurs and then becomes almost constant and equal to $\mu_{2}\left(\tilde{s}_{2}\right) / \alpha_{i}$. Then, after time $t=100$ days, the controller is turned off and the dilution rate is kept constant with no consequence for the digester, as the VFA concentration stays almost constant: the ecosystem is settled and composed of the most efficient population in the standard operating mode, such that it became naturally stable around the desired equilibrium $\left(s_{2}=\tilde{s}_{2}\right)$. This result is of great importance and must be verified experimentally : the selective start-up strategy gives birth to a stable ecosystem involving bacterial species such that the natural steady state is exactly the requested one, without needing a control feedback anymore.
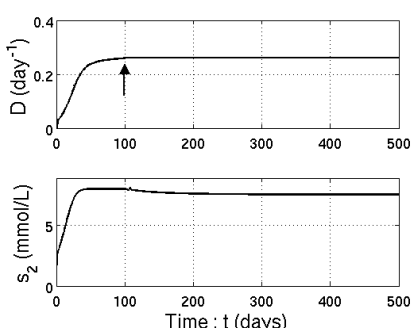

Fig. 6. The dilution rate (top) in the $\tilde{s}_{2}$ experiment was kept constant after time $t=100$ days with negligible consequence on the VFA concentration (bottom), showing that selection leads naturally to a consolidated ecosystem where the desired equilibrium $\left(s_{2}=\tilde{s}_{2}\right)$ is stable.

\section{APPLICATION TO A REAL ANAEROBIC DIGESTER START-UP PHASE}

In order to verify the proposed selection principle and to assess the effect of a controller that regulates the VFA during the start-up phase, experiments have been carried out at the LBE-INRA Laboratory in Narbonne (France).

\section{A. Experiment design}

The process is an up-flow anaerobic fixed bed reactor with a useful volume of $0.548 \mathrm{~m} 3$. The 
reactor is highly instrumented and many variables were measured during the experiments [9]. More details about the process and evaluation of its online instrumentation are available in [14].

\section{B. Experimental results and discussion}

Control (6) was applied, with command $\bar{s}_{2}=4$ $\mathrm{g} / \mathrm{L}$. The experimental results are presented in Fig. (7).
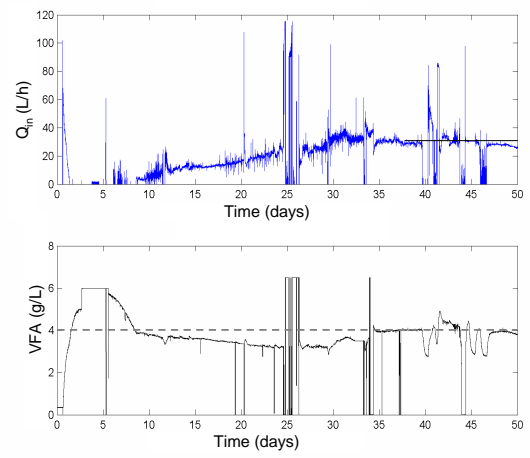

Fig. 7. Start-up experiment where $s_{2}$ is regulated at $\bar{s}_{2}=$ $4 g / L$. The influent flow rate $\left(Q_{i n}=D / V\right)$ and the VFA concentration are plotted.

We can see on this figure that our control objective was attained : the VFA concentration was well driven towards $\bar{s}_{2}$ despite many disturbances related to failures and disturbances in the process. It is worth noting that the more the ecosystem is selected, the more efficient the regulation. At the end, when the interesting species have probably been selected in the digester, the regulation is more and more efficient.

Finally, the proposed strategy enabled us to reach a high dilution rate $D=0.78$ while avoiding start up failure.

\section{CONCLUSIONS}

We proposed a start-up strategy for an anaerobic digester, whose aim is to influence the structure of the ecosystem, so that the obtained ecosystem in the end of start-up will be optimal for a given VFA concentration. For implementing such a selective process, we drive the competition by regulating the VFA concentration at a fixed value. We showed how the obtained ecosystem should also be consolidated because of the selection that occurs during the start-up phase.

According to model (1), a control law was proposed and a mathematical demonstration was given for predicting the competition outcome. Simulations were used to verify and explain the utility of the method, and a real experiment was presented where the start-up strategy was tested.
We emphasize that the aim of this strategy is not to minimize the start-up duration, but rather to build an efficient ecosystem. This kind of startup strategy, where the objective is to mould the structure of the bacterial community for optimizing a particular process, was never (to our knowledge) proposed before for anaerobic digestion, and further investigations in this direction are presently performed.

\section{ACKNOWLEDGMENTS}

This work has been carried out with the support provided by the CODA ARC project funded by the INRIA.

\section{REFERENCES}

[1] C. Delbès, R. Moletta, and J.-J. Godon, "Bacterial and archaeal 16s rdna and 16s rrna dynamics during an acetate crisis in an anaerobic digestor ecosystem," FEMS Microbiology Ecology, vol. 35, pp. 19-26, 2001.

[2] J. Hess and O. Bernard, "Design and study of a risk management criterion for an unstable anaerobic wastewater treatment process,' J. Process. Contr., vol. 18, pp. 71-79, 2008.

[3] K.-Y. how.and Y. Wang, S.-F. Foong, and J.-H.Tay, "Accelerated start-up and enhanced granulation in upflow anaerobic sludge blanket reactors," Water Research, vol. 28, pp. 2293-2304, 2004.

[4] S. Michaud, N. Bernet, P. Buffiére, M. Roustan, and R. Moletta, "Methane yield as a monitoring parameter for the strat-up of anaerobic fixed film reactors," Water Research, vol. 36, pp. 1385-1391, 2002.

[5] J. S. I. Ramirez, E.I.P. Volcke, "Modeling and monitoring of microbial diversity in ecosystems - application to biological wastewater treatment processes," in Proceedings of the IFAC conference. Seoul, Korea, 2008.

[6] H. L. Smith and P. Waltman, The theory of the chemostat: dynamics of microbial competition. Cambridge University Press, 1995.

[7] S. R. Hansen and S. P. Hubbell, "Single-nutrient microbial competition," Science, vol. 207, no. 28, pp. 1491-1493, 1980 .

[8] P. Masci, F. Grognard, and O. Bernard., "Continuous selection of the fastest growing species in the chemostat," in Proceedings of the IFAC conference. Seoul, Korea, 2008.

[9] O. Bernard, Z. Hadj-Sadok, D. Dochain, A. Genovesi, and J.-P. Steyer, "Dynamical model development and parameter identification for an anaerobic wastewater treatment process," Biotech.Bioeng., vol. 75, pp. 424-438, 2001.

[10] G. Bastin and D. Dochain, On-line estimation and adaptive control of bioreactors. Amsterdam: Elsevier, 1990.

[11] G. Wolkowicz and L. Zhiqi, "Global show, k.-y.; wang, y.; foong, s.-f.; tay, j.-h. accelerated start-up and enhanced granulation in upflow anaerobic sludge blanket reactors. water research 2004, 38, 2293-2304.dynamics of a mathematical model of competition in the chemostat: General response functions and differential death rates," SIAM Journal of Applied Mathematics, vol. 52, pp. 222-233, 1992.

[12] O. Bernard, Z. Hadj-Sadok, M. Pengov, and D. Dochain, "Modelling, monitoring and control of anaerobic digestion processes," Journal A., vol. 41, pp. 82-88, 2000.

[13] L. Mailleret, O. Bernard, and J.-P. Steyer, "Robust nonlinear adaptive control for bioreactors with unknown kinetics," Automatica, vol. 40:8, pp. 365-383, 2004.

[14] J. P. Steyer, J. C. Bouvier, T. Conte, P. Gras, and P. Sousbie, "Evaluation of a four year experience with a fully instrumented anaerobic digestion process," Water Science and Technology, vol. 45, pp. 495-502, 2002. 